\title{
Growth of two-dimensional Janus MoSSe by a single in situ process without initial or follow-up treatments
}

\author{
Chan Wook Jang', Won Jun Lee', Jae Kuk Kim', Sang Minh Park', Sung Kim² and Suk-Ho Choi (1)
}

\begin{abstract}
Two-dimensional (2D) Janus transition metal dichalcogenides (TMDCs) are highly attractive as an emerging class of 2D materials, but only a few methods are available for fabricating them. These methods rely on the initial growth of 2D TMDCs in one process, followed by an additional plasma or high-temperature (T) process. To overcome these drawbacks, we employ the new approach of $\mathrm{NaCl}$-assisted single-process chemical vapor deposition, which consists of three steps that proceed only by altering the temperature in situ. In the first step, $\mathrm{MoS}_{2}$ is deposited onto a $\mathrm{SiO}_{2} / \mathrm{Si}$ substrate with the $\mathrm{Mo}$ and $\mathrm{S}$ atoms activated in different temperature zones. In the second step, $\mathrm{S}$ vacancies are formed in the upper layer of the grown $\mathrm{MoS}_{2}$ by annealing. In the third step, the vacancies are filled with activated Se atoms. Throughout the steps, $\mathrm{NaCl}$ lowers the melting point of the constituent atoms, while the $\mathrm{T}$ in each zone is properly controlled. The growth mechanism is clarified by a separate annealing experiment that does not involve a supply of activated atoms. These results highlight a simple and cost-effective approach for growing Janus MoSSe, which is more useful for fundamental studies and device applications.
\end{abstract}

\section{Introduction}

Two-dimensional (2D) Janus transition metal dichalcogenides (TMDCs) have recently attracted increasing attention as an emerging class of $2 \mathrm{D}$ materials. The transition metal layer of Janus 2D TMDCs is sandwiched by two different chalcogen layers, resulting in broken mirror symmetry along the out-of-plane direction, which distinguishes Janus 2D TMDCs from pristine TMDCs. This unique characteristic of 2D Janus TMDCs leads to their novel phenomena, such as Rashba spin splitting, piezoelectric polarization, second-harmonic generation, catalytic effects, and long carrier recombination ${ }^{1-7}$, which are very promising for their application in sensors,

\footnotetext{
Correspondence: Suk-Ho Choi (sukho@khu.ac.kr)

1 Department of Applied Physics, Institute of Natural Sciences, and Integrated Education Institute for Frontier Science and Technology (BK21 Four), Kyung Hee University, Yongin 17104, Korea

${ }^{2}$ Humanitas College, Department of Physics, and Integrated Education Institute for Frontier Science and Technology (BK21 Four), Kyung Hee, University, Yongin 17104, Korea

These authors contributed equally: Chan Wook Jang, Won Jun Lee.
}

actuators, piezo/thermal-electric devices, solar cells, ion batteries, and other electromechanical devices ${ }^{8-11}$. The asymmetry of Janus TMDCs has also been used to tailor the van der Waals (vdW) interlayer coupling and charge transfer in vdW heterostructures ${ }^{12-15}$; such tailoring of Janus $\mathrm{MoSSe} / \mathrm{MoS}_{2}$ heterobilayers was performed by tuning the twist angle and interface composition ${ }^{16}$. The energy of graphene plasmons in vdW Janus MoSSe/graphene heterostructures was engineered up to $0.5 \mathrm{eV}$ by varying the number of Janus MoSSe layers ${ }^{17}$.

The synthesis technology of 2D Janus TMDCs has not yet been established, but recently, monolayer (ML) Janus MoSSe was successfully grown by controllably substituting $\mathrm{S}$ or Se atoms on the surface of a $\mathrm{MoS}_{2}$ or $\mathrm{MoSe}_{2}$ layer with Se or S atoms, respectively, based on two major approaches ${ }^{18,19}$. It is common for both materials, $\mathrm{MoS}_{2}$ and $\mathrm{MoSe}_{2}$, to be grown as a ML in a separate process. In one approach, the top $\mathrm{S}$ layer in the pregrown $\mathrm{MoS}_{2} 2 \mathrm{D}$ sheet was stripped and replaced with $\mathrm{H}$ atoms, and the $\mathrm{H}$ atoms were then thermally selenized under vacuum ${ }^{18}$. In

\section{c The Author(s) 2022}

(c) Open Access This article is licensed under a Creative Commons Attribution 4.0 International License, which permits use, sharing, adaptation, distribution and reproduction cc) in any medium or format, as long as you give appropriate credit to the original author(s) and the source, provide a link to the Creative Commons license, and indicate if changes were made. The images or other third party material in this article are included in the article's Creative Commons license, unless indicated otherwise in a credit line to the material. If material is not included in the article's Creative Commons license and your intended use is not permitted by statutory regulation or exceeds the permitted use, you will need to obtain permission directly from the copyright holder. To view a copy of this license, visit http://creativecommons.org/licenses/by/4.0/. 
another approach, the top layer of Se in the pregrown $\mathrm{MoSe}_{2}$ ML was sulfurized by a controlled substitution reaction with vaporized $S^{19}$. The drawback of these methods is that they rely on follow-up treatments in addition to the initial chemical vapor deposition (CVD) of $2 \mathrm{D} \mathrm{MoS}_{2}$ or $\mathrm{MoSe}_{2}$ TMDCs, which can result in increased complexity and cost. To overcome these limitations, we developed a new method of Janus MoSSe growth, simplified by employing a single $\mathrm{NaCl}$-assisted process in a CVD apparatus. This approach involves three-step processes in situ to sequentially activate Mo and $S$ for the growth of $\mathrm{MoS}_{2}$, form S vacancies in the top $\mathrm{MoS}_{2}$ layer, and activate Se to fill the vacancies only by altering the temperature $(\mathrm{T})$ without using any other means, such as $\mathrm{H}_{2}$ plasma ${ }^{18}$. Throughout the steps, the temperature in each chalcogen and transitional-metal zone was properly controlled, and $\mathrm{NaCl}$ lowered the melting point of the material. The grown Janus MoSSe was systemically examined by Raman scattering, photoluminescence (PL), atomic/Kelvin-probe force microscopy (AFM and KPFM), and optical microscopy (OM). Based on the experimental results, a possible growth mechanism is proposed. These results are believed to provide a simple, cost-effective, and universal approach for growing 2D Janus TMDCs; this approach is more useful for fundamental studies and device applications.

\section{Materials and methods}

Triangle-shaped 2D flakes were produced on $300 \mathrm{~nm}$ $\mathrm{SiO}_{2} / \mathrm{Si}$ wafers by the $\mathrm{NaCl}$-assisted vaporization of a mixture of $\mathrm{MoO}_{3}, \mathrm{~S}$ (or Se), and $\mathrm{NaCl}$ powders in a quartz tube furnace under a controlled gaseous environment. $\mathrm{NaCl}$ in the growth process was found to play a vital role in lowering the growth $\mathrm{T}^{20,21}$. A typical run of growth for $\mathrm{MoS}_{2}$ was performed by loading $15 \mathrm{mg}$ $\mathrm{MoO}_{3}+20 \mathrm{mg} \mathrm{NaCl}$ powder in one boat in the high- $\mathrm{T}$ zone and $35 \mathrm{mg} \mathrm{S}$ powder in the other boat in the low- $\mathrm{T}$ zone within the same quartz tube. A piece of the $\mathrm{SiO}_{2} / \mathrm{Si}$ substrate was placed at the downstream location close to the high- $\mathrm{T}$ boat. The low- and high- $\mathrm{T}$ zones in the furnace were heated to 150 and $750{ }^{\circ} \mathrm{C}$, respectively, for $10 \mathrm{~min}$; they were kept at these temperatures for $10 \mathrm{~min}$ for growing $\mathrm{MoS}_{2}$. The vapor-phase reactants were transported by a mixed gas of $\mathrm{H}_{2}$ and $\mathrm{Ar}$, thereby facilitating the growth of the 2D crystals in the growth region. The flow rate of $\mathrm{H}_{2}$ gas was fixed at $20 \mathrm{sccm}$. All the reactions were carried out at $10^{-1}$ Torr, and their heating rate was $25^{\circ} \mathrm{C} \mathrm{min}{ }^{-1}$. At the end of the growth phase, the furnace was left to naturally cool to room temperature. The procedure for synthesizing Janus MoSSe is detailed in the next section. To optimize the growth of Janus MoSSe, the distance between the boats and the rate of Ar gas were varied from 11 to $17 \mathrm{~cm}$ and 82 to $88 \mathrm{sccm}$, respectively.
Raman scattering and PL measurements were carried out using a confocal Raman/PL microscope (HEDA, NOST, Korea) with a $532 \mathrm{~nm}$ laser. A laser with a typical incident power of $100 \mu \mathrm{W}$ was linearly polarized and focused to a spot size of $<1 \mu \mathrm{m}$ by a $100 \times$ objective lens (numerical aperture, 0.9). The sample was mounted on a piezo-stepper table and scanned under the microscope. The spatial resolution of this setup was approximately $500 \mathrm{~nm}$. The PL emission (or Raman scattering) signal was collected with the same objective, dispersed with a monochromator, and detected using a charge-coupled device (Andor Technology). The Raman scattering behaviors of MoSSe and $\mathrm{MoSe}_{2}$ were also measured as a function of the polarization angle by employing a linear polarizer. The KPFM surface potential profiles and mapping images were obtained in the noncontact mode of an atomic force microscope (Park Systems model XE-100). The samples were transferred to indium tin oxide (ITO)coated glasses for the KPFM measurements to prevent the problem of surface charging. The I-V curves were also obtained on ITO substrates in the conductive mode of the atomic force microscope.

\section{Results and discussion}

Figure 1a and $\mathrm{b}$ show the CVD apparatus for growing Janus MoSSe and a schematic diagram explaining the growth mechanism, respectively. The CVD system is divided into two zones, the low- and high- $T$ zones, referred to as zones 1 and 2, respectively. The Se and S powders are located at separate positions in one boat, which is located in zone 1 , while the other boat containing $\mathrm{MoO}_{3}+\mathrm{NaCl}$ powder and $\mathrm{SiO}_{2} / \mathrm{Si}$ substrate is located in zone 2. In the first step, a $\mathrm{MoS}_{2}$ flake is synthesized on a $\mathrm{SiO}_{2} / \mathrm{Si}$ substrate, where the Mo and $\mathrm{S}$ atoms are activated at $T=750$ and $150^{\circ} \mathrm{C}$, respectively, in each zone (Supplementary Fig. S1). The grown $\mathrm{MoS}_{2}$ flake showed typical Raman and PL spectra peaked at $382.6 / 406.4 \mathrm{~cm}^{-1}$ $\left(E_{2 g} / A_{1 g}\right.$ modes) and $691 \mathrm{~nm}$, respectively (Fig. 2a and b), as previously reported ${ }^{22-24}$. The resulting difference between the Raman peaks was calculated to be $23.8 \mathrm{~cm}^{-1}$ and corresponded to a triple-layer $\mathrm{MoS}_{2}{ }^{25}$. In the second step, $\mathrm{S}$ vacancies were formed in the $\mathrm{MoS}_{2}$ flake (Fig. 1b), while the $\mathrm{T}$ in zone 2 was changed to $580^{\circ} \mathrm{C}$ for $10 \mathrm{~min}$ without the addition of any activated atoms. This formation of vacancies was demonstrated in a separate annealing experiment, as described below. In the third step, the $\mathrm{S}$ vacancies in the top-surface $\mathrm{MoS}_{2}$ were filled with Se atoms activated at $T=300{ }^{\circ} \mathrm{C}$ (Fig. $1 \mathrm{~b}$ and Supplementary Fig. S1) to finally form Janus MoSSe. The vacancies were believed to be formed concomitantly in the layer during this last step, as was also demonstrated by the annealing experiment.

The growth of the Janus MoSSe layers was optimized through variation of the principal deposition parameters. 
(a)

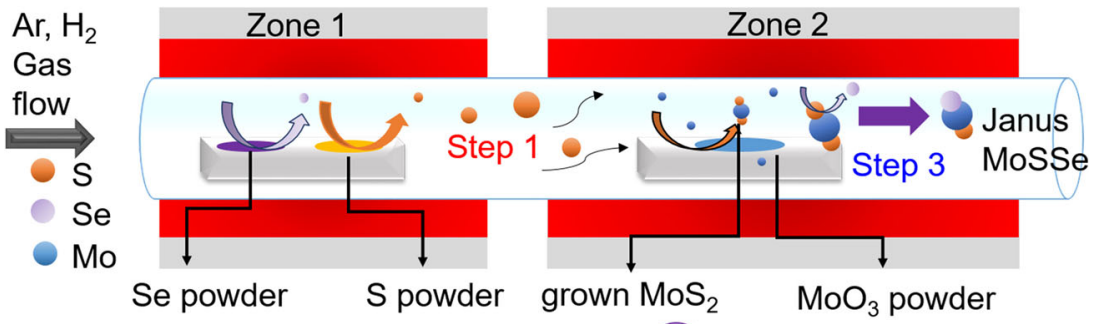

(b)

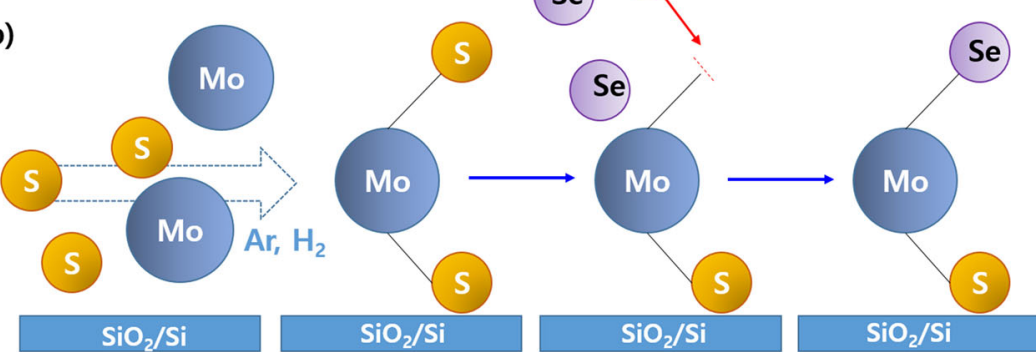

Fig. 1 Growth of Janus MoSSe. a CVD apparatus for Janus MoSSe. The CVD system is divided into two zones, zones 1 and 2. One boat for the Se and $\mathrm{S}$ powders is located in zone 1, while the other boat for the $\mathrm{MoO}_{3}+\mathrm{NaCl}$ powder and the $\mathrm{SiO}_{2} / \mathrm{Si}$ substrate is located in zone 2 . The Se and $\mathrm{S}$ powders are separated within the boat. $\mathbf{b}$ Schematic diagram detailing the growth mechanism. The in situ single growth process for MosSe consists of three steps: synthesis of $\mathrm{MoS}_{2}$ flakes, formation of S vacancies on the top-surface $\mathrm{MoS}_{2}$ layer, and growth of Janus MoSSe.

During this procedure, the shape, size, and uniformity of the Janus MoSSe were shown to be very sensitive to a few major parameters, such as the distance (d) between the boats and the Ar flow rate $\left(\mathrm{R}_{\mathrm{Ar}}\right)$. Figure 3 shows $\mathrm{OM}$ images of the flakes; these images were obtained by varying $d$ at a fixed $R_{A r}$ of $86 \mathrm{sccm}$ (see Supplementary Fig. $S 2$ for the optimization through the variation of $R_{A r}$ at a fixed $\mathrm{d}$ of $15 \mathrm{~cm}$ ) and the corresponding Raman and PL spectra. These results suggest that the Janus MoSSe with the best quality was fabricated under the optimized conditions: $\mathrm{d}=15 \mathrm{~cm}$ and $\mathrm{R}_{\mathrm{Ar}}=86 \mathrm{sccm}$. As shown in the OM image of the optimized flake (Fig. 4a), the inner and outer regions are clearly distinguished by their triangular boundary between the layers. The Raman spectrum in the inner (or the outer) region exhibits major peaks at 287.2/ 350.2 (or $239.6 / 287.3) \mathrm{cm}^{-1}$, corresponding to the $\mathrm{A}_{1 \mathrm{~g}} / \mathrm{E}_{2 \mathrm{~g}}$ modes of MoSSe (or $\mathrm{MoSe}_{2}$ ), respectively ${ }^{18,19,26}$, as shown in Fig. 3e (or Fig. 2a). The difference between the $A_{1 g}$ and $E_{2 \mathrm{~g}}$ modes of MoSSe is calculated to be approximately 63 $\mathrm{cm}^{-1}$, which is very close to the theoretical and experimental values $\left(\sim 60 \mathrm{~cm}^{-1}\right)$ reported for a MoSSe monolayer ${ }^{19,27,28}$. Here, it should be noted that the $\mathrm{MoS}_{2}$ peaks are also visible in the Raman spectrum of the inner region, indicating that the $\mathrm{MoS}_{2}$ layer remains at the bottom of the Janus MoSSe even after optimization, resulting in the actual formation of a MoSSe/ $\mathrm{MoS}_{2}$ heterostructure.

Figures $2 \mathrm{~b}$ and $3 \mathrm{f}$ show PL spectra of the inner and outer regions of the optimized flake. These spectra have peaks at 726 and $809 \mathrm{~nm}$, which are consistent with those of MoSSe and $\mathrm{MoSe}_{2}$, respectively ${ }^{18,19,26}$. As shown in the PL mapping images (Fig. $4 \mathrm{~b}-\mathrm{c}$ ), the variation in the peak wavelength is clearly observed as the position moves in the direction from the inner region, the interface, to the outer region. This further demonstrates that the Janus MoSSe inside the flake is surrounded by the peripheral $\mathrm{MoSe}_{2}$ region with the boundary between the regions. Near the boundary of the inner/outer regions, the intermediate PL and Raman spectra exhibited peaks corresponding to both MoSSe and $\mathrm{MoSe}_{2}$ (Supplementary Fig. S3), resulting from the mixed phases of MoSSe and $\mathrm{MoSe}_{2}$ near the boundary. As the position moves in the inner-toouter region direction at the boundary, the Raman and PL peak intensities of MoSSe decrease, while those of $\mathrm{MoSe}_{2}$ increase. Within the inner region, the PL peak redshifts in the center-to-edge direction (Fig. 4c).

Figure $4 \mathrm{~d}-\mathrm{g}$ and $\mathrm{e}-\mathrm{h}$ compare the KPFM mapping images/surface potential profiles and AFM height profiles of $\mathrm{MoS}_{2}$ and MoSSe. As shown in Fig. 4g, the potential profile of $\mathrm{MoS}_{2}$ looks very uniform in the full region and is clearly differentiated from that of the substrate. Figure $4 \mathrm{~h}$ shows a considerable change in the potential due to the formation of Janus MoSSe with respect to the substrate, together with a potential dip in the peripheral $\mathrm{MoSe}_{2}$ region, consistent with the PL mapping images in Fig. 4b-c. The polarization-angle dependences of the Raman behaviors were measured for the polar plots of the peak intensities in the inner (MoSSe) and outer $\left(\mathrm{MoSe}_{2}\right)$ regions (Supplementary 

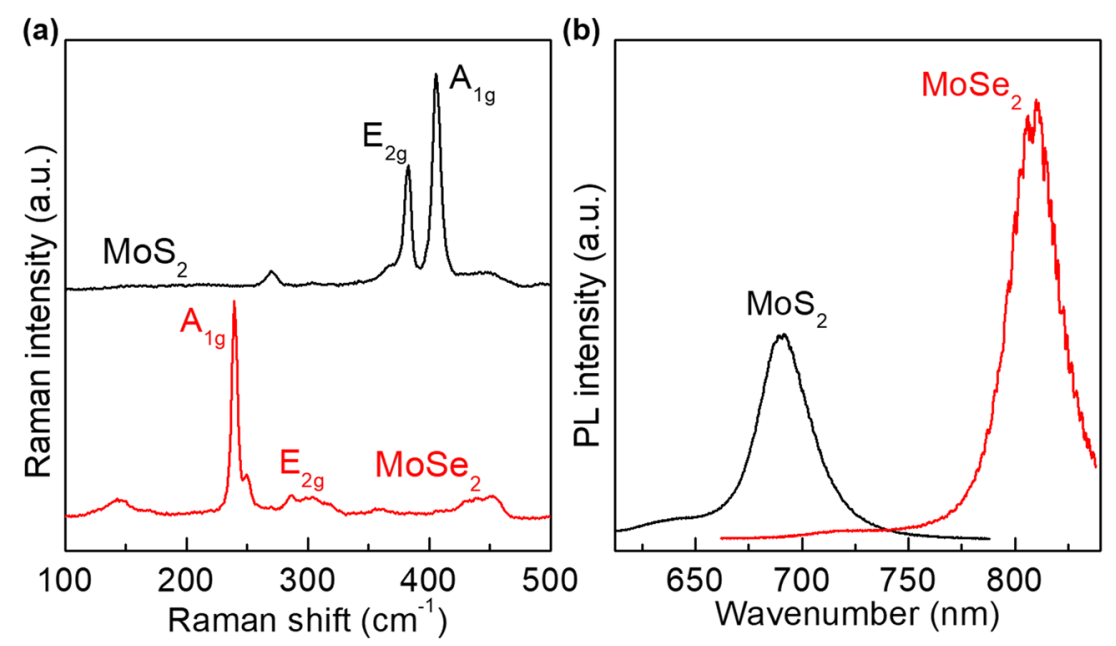

Fig. 2 Raman and PL spectra. a Raman and $\mathbf{b}$ PL spectra of the $\mathrm{MoS}_{2}$ flake grown in the first step and the MoSe $e_{2}$ in the peripheral region of the flake removed after the whole steps for the formation of the Janus MoSSe were finished. These spectra are consistent with those of $\mathrm{MoS}_{2}$ and $\mathrm{MoSe}_{2}$ reported in previous publications.
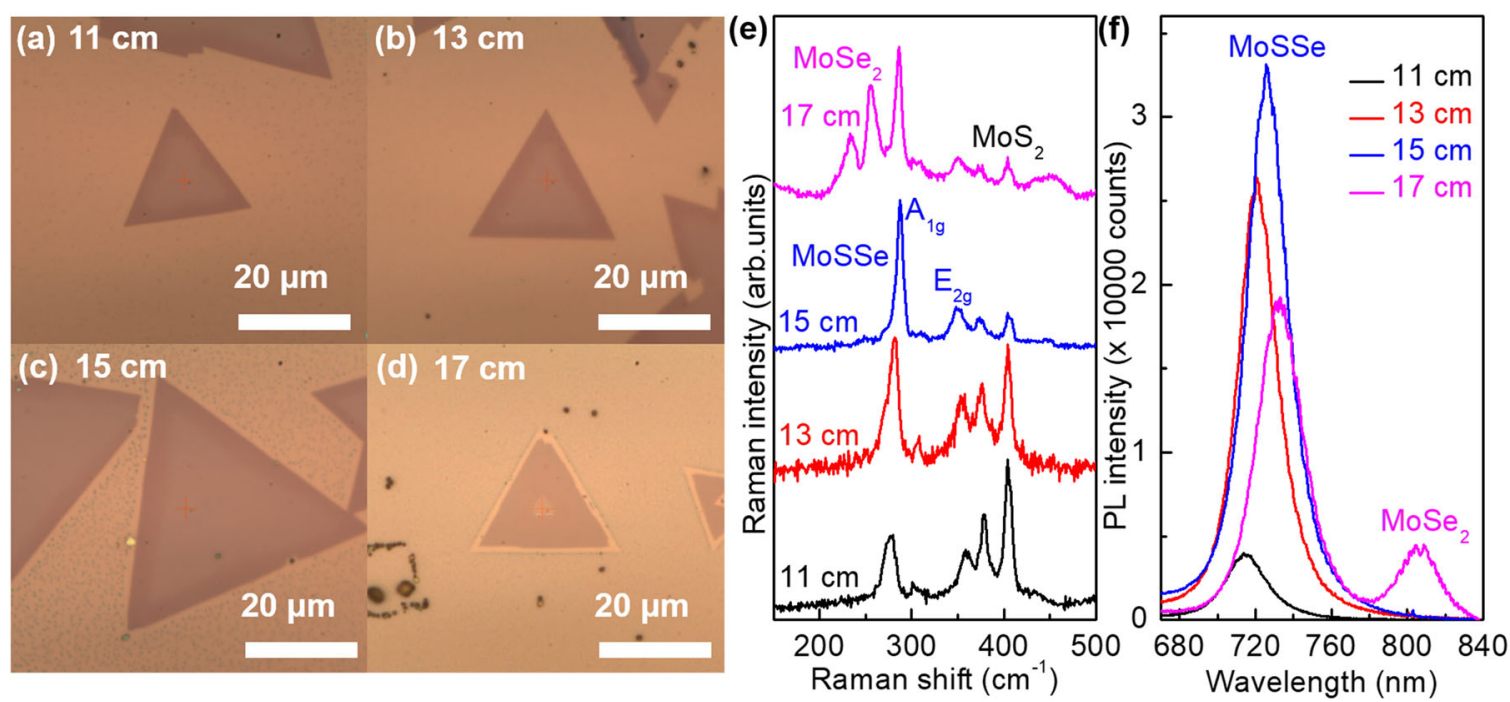

Fig. 3 Optimization of Janus MoSSe. a-d Optical microscopy images showing the Janus MoSSe flakes obtained by varying the distance between the boats in zones 1 and 2 from 11 to $17 \mathrm{~cm}$, with the Ar flow rate fixed at $86 \mathrm{sccm}$ for optimization, and e-f corresponding Raman and PL spectra (see Supplementary Fig. S2 for optimization by varying the Ar flow at a fixed d of $15 \mathrm{~cm}$ ). These results suggest that the Janus MoSSe is optimized when the distance between the boats $=15 \mathrm{~cm}$ and the Ar rate $=86 \mathrm{sccm}$ in this growing system.

Fig. S4). According to the Raman selection rules based on symmetry, the intensity of the $E_{2 g}$ mode was predicted to be independent of the polarization angle, while that of the $\mathrm{A}_{1 \mathrm{~g}}$ mode was predicted to be polarized following the formula $I_{\mathrm{A} 1 \mathrm{~g}}=A \cos ^{2} \theta$, where $I_{\mathrm{A} 1 \mathrm{~g}}$ is the intensity, $A$ is a constant, and $\theta$ is the angle ${ }^{29}$. This reasoning explains why the line shapes of the $\mathrm{A}_{1 \mathrm{~g}}$ and $E_{2 g}$ modes were typical dumbbell and circular structures in the polar plots. Considerable differences in the polar plots between the MoSSe and $\mathrm{MoSe}_{2}$ regions were not observed, and this result was consistent with those of previous reports $^{29,30}$.

To analyze the conversion of $\mathrm{MoS}_{2}$ to MoSSe, a separate annealing process was performed by keeping a grown $\mathrm{MoS}_{2}$ flake at $580^{\circ} \mathrm{C}$ (same $\mathrm{T}$ as was used for the growth of MoSSe) for $10 \mathrm{~min}$ in zone 2 without atomic activation under Ar flow. After annealing, almost no difference in the Raman spectrum of the $\mathrm{MoS}_{2}$ flake was observed, but its PL spectrum was slightly redshifted (Supplementary Information, Fig. S5), which was consistent with a 

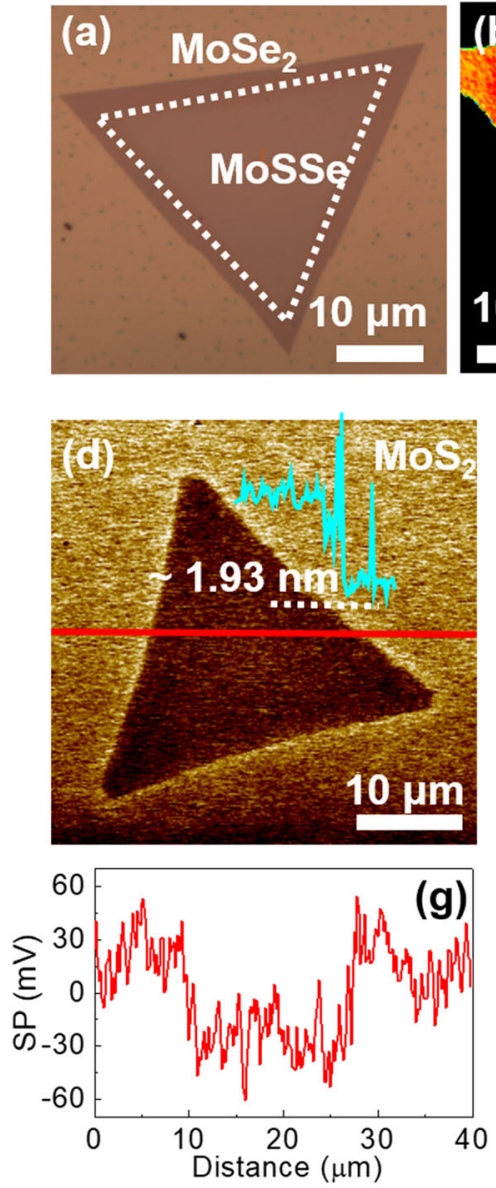

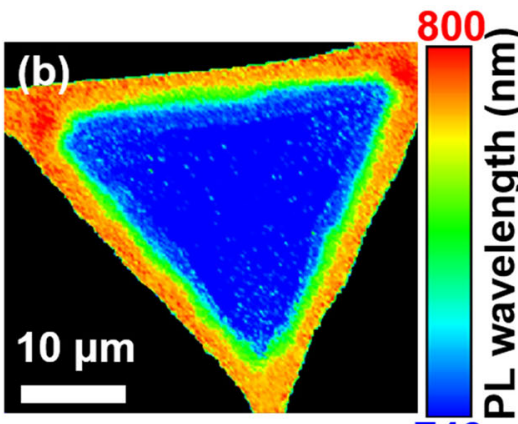

746
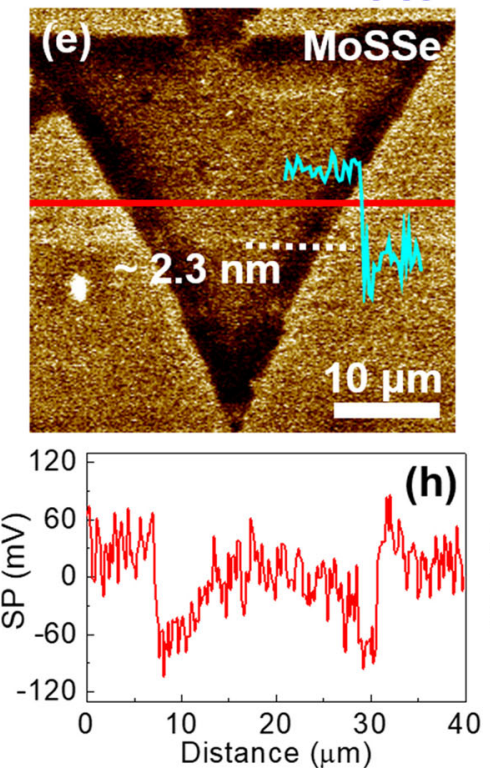
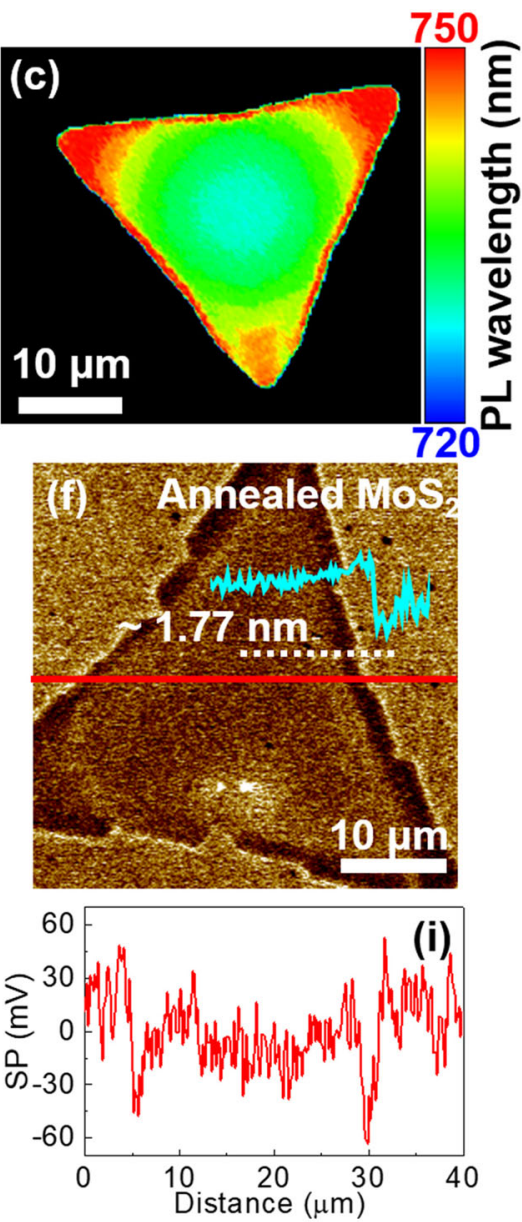

Fig. 4 Mapping images and height/potential profiles. a Optical microscopy and $\mathbf{b}$ PL peak-wavelength mapping images showing clear delineation between the inner Janus MoSSe and the outer $\mathrm{MoSe}_{2}$ regions. c PL peak-wavelength mapping image showing position-dependent variation of the peak wavelength within the inner Janus MoSSe region. The variation in the peak wavelength is clearly observed as the position moves in the direction from the inner region, the interface, to the outer region. Within the inner region, the PL peak redshifts in the center-to-edge direction, possibly resulting from the position-dependent sequential variation of the $\mathrm{S}$ vacancies and/or the occupation of the Se atoms in the vacancies. KPFM images and corresponding surface potential profiles of $\mathbf{d}-\mathbf{g}$ as-grown $\mathrm{MoS}_{2}$, e-h Janus MoSSe, and $\mathbf{f}-\mathbf{i}$ annealed MoS $\mathbf{S}_{2}$ flakes. Here, the surface potential profiles were obtained following the red lines drawn on the KPFM images, and the AFM height profiles of the three flakes are indicated on the KPFM images.

previous report ${ }^{31}$. Figure $4 \mathrm{~d}-\mathrm{g}$ and $\mathrm{f}-\mathrm{i}$ compare the KPFM mapping images and surface potential profiles of the grown and annealed $\mathrm{MoS}_{2}$ flakes. A large change in the potential of $\mathrm{MoS}_{2}$, including a sharp drop at the edge, was observed with respect to the substrate during annealing, as shown in Fig. 4i. In other words, the $\mathrm{MoS}_{2}$ flake was fully changed by annealing, with the inner and peripheral parts being structurally different. No correlations between the KPFM results and the surface morphologies were observed, as confirmed by the AFM images (Supplementary Fig. S6). In addition, several vacant regions were identified in the annealed $\mathrm{MoS}_{2}$ flake by OM and PLmapping images (Supplementary Information, Fig. S7) and can be regarded as $\mathrm{S}$ vacancies, as previously repor$\operatorname{ted}^{31-33}$. These results demonstrate the formation of $S$ vacancies in the $\mathrm{MoS}_{2}$ flake before and during the addition of activated Se (2nd \& 3rd steps) in the fabrication process, as described above.

Mono- and bilayer $\mathrm{MoS}_{2}$ flakes were also grown in the 1st step of Janus MoSSe fabrication, but after the 2nd and 3rd steps, no Janus MoSSe had formed, possibly due to the excessive thermal budget of these steps. This result further suggests that there is a critical minimum thickness for the successful growth of Janus MoSSe using our approach. Similar behaviors were reported in previous publications ${ }^{33,34}$. In these reports, the mono-layer $\mathrm{MoS}_{2}$ was cracked when annealed above a critical temperature (this temperature depends on the approach). We also examined the samples after the 2nd step (similar to annealing); these samples included the mono- and bilayer 
$\mathrm{MoS}_{2}$ flakes grown in the 1st step. Almost no monolayer material remained, while for the bilayer, some $\mathrm{MoS}_{2}$ debris was scattered on the $\mathrm{SiO}_{2} / \mathrm{Si}$ surface (Supplementary Information, Fig. S8). To solve this problem, our system should be redesigned to reduce the excessive thermal budget, especially for the cases of the mono- or bilayer $\mathrm{MoS}_{2}$. In contrast, the thermal load in thicker samples with $\geq 3$ layers seems to be well distributed throughout the whole volume. The thick $\mathrm{MoS}_{2}$ layer may also protect the Mo-S bond at the bottom. In addition, $\mathrm{MoSe}_{2}$ formed in the peripheral region of the flake could act as a thermal barrier to reduce the thermal budget of the bottom Mo-S bond. This result explains why the breakage of the Mo-S bond at the surface was described when vacancies formed in the growth mechanism (Fig. 1b). If the annealing time and/or temperature is longer, even the bottom Mo-S bond will break. The optimum time and temperature for the Janus MoSSe were obtained after much trial and error.

The uniformity in the region of the Janus MoSSe was evaluated by measuring the PL and Raman spectra at different positions in the direction from the center to the vertex within the inner region of the triangular flake, as shown in Fig. 5a. Figure 5b, c show the positiondependent Raman spectra of MoSSe and $\mathrm{MoS}_{2}$ in the inner region and the corresponding shifts of the $A_{1 g} / E_{2 g}$ peak wavenumbers. Here, no considerable changes in the Raman peak energies were observed, as shown in Fig. 5c, but the main Raman peak ratio of $\mathrm{MoSSe} / \mathrm{MoS}_{2}$ decreased from the center to the vertex, as shown in Fig. 5b (and Supplementary Fig. S9a). The PL peak of MoSSe redshifted in the same direction, as shown in Figs. 5d and 4c (and Supplementary Fig. S9b), and this result is very consistent with a previous report ${ }^{33}$. These results are possibly due to the position-dependent sequential variation of the $S$ vacancies and/or the occupation of the Se atoms in the vacancies, as previously reported ${ }^{31,32}$.

It is widely known that the crystalline structure is almost unchanged by the $\mathrm{MoS}_{2} \rightarrow$ MoSSe transformation, as shown in various publications ${ }^{18,19}$. The Janus MoSSe remains in a $2 \mathrm{H}$ crystalline phase even by the top layer $\mathrm{S}$ (or Se)-atom substitution of Se (or S) in $\mathrm{MoSe}_{2}\left(\right.$ or $\mathrm{MoS}_{2}$ ), indicating that there is neither significant lattice distortion nor phase transition. Additionally, according to our results, the crystalline structure of the Janus MoSSe flake remained stable but its shape and size changed depending on the preparation conditions (Fig. 3 and Supplementary Fig. S2), as confirmed by the PL and Raman spectra. Even though other phases of Janus MoSSe, such as $1 \mathrm{~T}$ or $1 \mathrm{~T}^{\prime}$ exist, PL cannot be observed due to the metallic properties of the $1 \mathrm{~T}$ or $1 \mathrm{~T}^{\prime}$ phases, and their Raman spectra differ from that of the $2 \mathrm{H}$ phase, as shown in other $2 \mathrm{D}$ materials.

The shape of the 2D flakes is known to strongly depend on the preparation conditions ${ }^{35-37}$, but most of the $2 \mathrm{D}$ flakes are predominantly triangle-shaped. For example, the crystal shape of the $\mathrm{MoS}_{2}$ at equilibrium is hexagonal under Mo-rich conditions, while it becomes triangular under S-rich conditions ${ }^{36}$. More importantly, constructing larger and more uniform Janus crystals with the smallest possible defect density is a preeminent challenge. We accidentally succeeded in making Janus MoSSe with almost no peripheral $\mathrm{MoSe}_{2}$ region (Supplementary Fig. S10), even though its preparation conditions needed to be further optimized for stabilization. In contrast, more $\mathrm{S}$ vacancies tended to form in hexagonal $\mathrm{MoS}_{2}$ shapes, and their distributions gradually varied from the vertices and edges (triangular shape) to the center (hexagonal shape) $)^{37}$. Based on these findings, we focused on trying to make the Janus flakes as large and uniform as possible to secure a larger central MoSSe region in each triangular flake.

The I-V curves measured for the inner MoSSe and outer $\mathrm{MoSe}_{2}$ regions showed rectifying behaviors, with steeper voltage-dependent current variation in the $\mathrm{MoSe}_{2}$ region (Supplementary Fig. S11). Both layers formed heterojunctions with the bottom $\mathrm{MoS}_{2}$ layer. The work function of $\mathrm{MoS}_{2}$ was measured to be $-4.85,-4.96,-5.09$, and $-5.13 \mathrm{eV}$ for layer numbers $1,2,4$, and 8 , respectively, as was noted in our previous report ${ }^{38}$. Therefore, the work function of the bottom $\mathrm{MoS}_{2}$ layer was estimated to be approximately $-5.0 \mathrm{eV}$. The work function of monolayer $\mathrm{MoSe}_{2}$ is known to be $4.70 \mathrm{eV}^{39}$. The heterojunction of Janus MoSSe with $\mathrm{MoS}_{2}$ is classified into two types: S/S and $\mathrm{Se} / \mathrm{S}$ heterostructures ${ }^{16}$. The former corresponds to our case because the Se layer is at the top of the MoSSe. The work function of MoSSe in the S/S heterostructure was calculated to be $-4.26 \mathrm{eV}^{16}$. Based on these considerations, the barrier height of the $\mathrm{MoSe}_{2} / \mathrm{MoS}_{2}$ junction is lower than that of the MoSSe/MoS 2 junction, and this conclusion is consistent with the I-V behavior. These results are preliminary, so further in-depth characterization is needed to clarify the device properties of Janus MoSSe in future work.

\section{Conclusion}

We have shown the successful fabrication of Janus MoSSe by a new simplified method that employs a single $\mathrm{NaCl}$-assisted process in a CVD apparatus. This approach involved three-step processes to sequentially activate Mo and $\mathrm{S}$ in situ to grow $\mathrm{MoS}_{2}$, form S vacancies in the topsurface $\mathrm{MoS}_{2}$ layer, and activate Se to fill the vacancies by only altering the temperature. The $\mathrm{MoS}_{2}$ layer was shown to remain at the bottom of the Janus MoSSe even after optimization, resulting in the formation of a MoSSe/MoS heterostructure. Throughout these steps, $\mathrm{NaCl}$ helped lower the melting points of the constituent substances, while the $\mathrm{T}$ in each zone was properly controlled. A possible growth mechanism was proposed based on the results of another annealing experiment that did not 

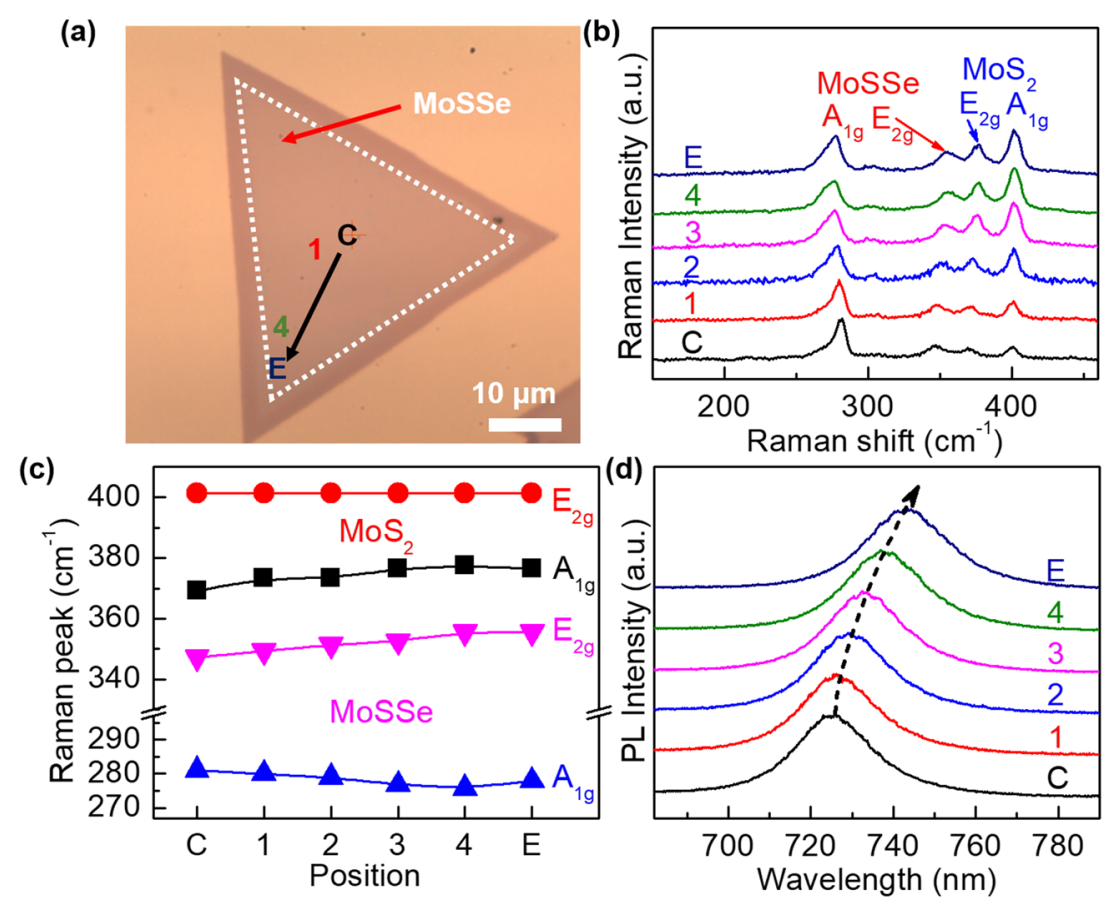

Fig. 5 Position dependence within Janus MoSSe. a Optical microscopy image showing the direction of the position movement from the center to the edge within the inner Janus MoSSe region for Raman and PL measurements. b-d Position-dependent Raman spectra, shifts of the Raman peaks, and PL spectra. Here, any considerable position dependence in the Raman shifts is invisible, but as the position moves in the direction from the center to the vertex, the intensity ratio of the MoSSe/MoS Raman A 1 peaks decreases, and the PL peak redshifts (see also Supplementary Fig. S9).

supply activated atoms. This simple approach may open an easy way to fabricate various kinds of $2 \mathrm{D}$ Janus materials, which are very promising for their fundamental characteristics and applications in novel devices.

\section{Acknowledgements}

This work was supported by a National Research Foundation of Korea (NRF) grant funded by the Korean government (MSIT) (No. 2020R1A2C2004376).

\section{Author contributions}

C.W.J. and W.J.L. prepared the 2D flakes and carried out the Raman and PL measurements. C.W.J. characterized the flakes by AFM and KPFM. J.K.K. performed the annealing experiments. S.M.P. obtained optical images of the flakes. S.K. analyzed the Raman and PL data. S.-H.C. initiated and supervised the work and wrote the paper. All authors discussed the results and commented on the manuscript.

\section{Conflict of interest}

The authors declare no competing interests.

\section{Publisher's note}

Springer Nature remains neutral with regard to jurisdictional claims in published maps and institutional affiliations.

Supplementary information The online version contains supplementary material available at https://doi.org/10.1038/s41427-022-00363-x.

Received: 17 October 2021 Revised: 12 January 2022 Accepted: 20 January 2022.

Published online: 25 February 2022

\section{References}

1. Yagmurcukareds, M. et al. Quantum properties and applications of 2D Janus crystals and their superlattices. Appl. Phys. Rev. 7, 011311 (2020).

2. Li, R., Cheng, Y. \& Huang, W. Recent progress of Janus $2 \mathrm{D}$ transition metal chalcogenides: From theory to experiments. Small 14, 1802091 (2018).

3. Zhang, L. et al. Recent advances in emerging Janus two dimensional materials: from fundamental physics to device applications. J. Mater. Chem. A 8, 8813-8830 (2020).

4. Zheng, T. et al. Excitonic Dynamics in Janus MoSSe and WSSe Monolayers. Nano Lett. 21, 931-937 (2021).

5. Wei, Y., Tang, X., Shang, J., Ju, L. \& Kou, L. Two-dimensional functional materials: from properties to potential applications. Int. J. Smart Nano Mater. 11, 247-264 (2020).

6. Wang, J. et al. Intriguing electronic and optical properties of two-dimensional Janus transition metal dichalcogenides. Phys. Chem. Chem. Phys. 20, 18571-18578 (2018).

7. Han, L. et al. Anomalous behavior of $2 \mathrm{D}$ Janus excitonic layers under extreme pressures. Adv. Mater. 32, 2002401 (2020).

8. Ko, K. Y. et al. High-performance gas sensor using a large-area $\mathrm{WS}_{2 \times} \mathrm{Se}_{2-2 x}$ alloy for low-power operation wearable applications. ACS Appl. Mater. Interfaces 10, 34163-34171 (2018).

9. Liu, Y.-L., Shi, Y. \& Yang, C.-L. Two-dimensional MoSSe/g-GeC van der waals heterostructure as promising multifunctional system for solar energy conversion. Appl. Surf. Sci. 545, 148952 (2021).

10. Ju, L., Bie, M., Shang, J., Tang, X. \& Kou, L. Janus transition metal dichalcogenides: a superior platform for photocatalytic water splitting. J. Phys. Mater. 3, 022004 (2020).

11. Dong, L., Lou, J. \& Shenoy, V. B. Large in-plane and vertical piezoelectricity in Janus transition metal dichalchogenides. ACS Nano 11, 8242-8248 (2017).

12. Li, L. et al. Wavelength-tunable interlayer exciton emission at the near-infrared region in van der Waals semiconductor heterostructures. Nano Lett. 20 $3361-3368$ (2020) 
13. Le, C. T. et al. Effects of interlayer coupling and band offset on second harmonic generation in vertical $\mathrm{MoS}_{2} / \mathrm{MoS}_{2(1-x)} \mathrm{Se}_{2 x}$ structures. ACS Nano 14, 4366-4373 (2020).

14. Riis-Jensen, A. C., Pandey, M. \& Thygesen, K. S. Efficient charge separation in 2D Janus van der Waals structures with built-in electric fields and intrinsic $p-n$ doping. J. Phys. Chem. C. 122, 24520-24526 (2018).

15. Palsgaard, M., Gunst, T., Markussen, T., Thygesen, K. S. \& Brandbyge, M. Stacked Janus device concepts: Abrupt pn-junctions and cross-plane channels. Nano Lett. 18, 7275-7281 (2018)

16. Zhang, K. et al. Efthimios Kaxiras, Spectroscopic signatures of interlayer coupling in Janus MoSSe/MoS 2 heterostructures. ACS Nano 15, 14394-14403 (2021).

17. Cavalcante, L. S. R., Gjerding, M. N., Chaves, A. \& Thygesen, K. S. Enhancing and controlling plasmons in Janus MoSSe-Graphene based van der Waals heterostructures. J. Phys. Chem. C. 123, 16373-16379 (2019).

18. Lu, A.-Y. et al. Janus monolayers of transition metal dichalcogenides. Nat. Nanotechnol. 12, 744-749 (2017).

19. Zhang, J. et al. Janus monolayer transition-metal dichalcogenides. ACS Nano 11, 8192-8198 (2017).

20. Zhou, J. et al. A library of atomically thin metal chalcogenides. Nature 556, 355-359 (2018).

21. Wang, Z. et al. $\mathrm{NaCl}$-assisted one-step growth of $\mathrm{MoS}_{2}-\mathrm{WS}_{2}$ in-plane heterostructures. Nanotechnology 28, 325602 (2017).

22. Chen, $\mathrm{H}$. et al. Ultrafast formation of interlayer hot excitons in atomically thin $\mathrm{MoS}_{2} \mathrm{NS}_{2}$ heterostructures. Nat. Commun. 7, 12512 (2016).

23. Zhang, J. et al. Interlayer-state-coupling dependent ultrafast charge transfer in $\mathrm{MoS}_{2} \mathrm{NS}_{2}$ bilayers. Adv. Sci. 4, 1700086 (2017).

24. Pawbake, A. S., Pawar, M. S., Jadkar, S. R. \& Late, D. J. Large area chemical vapor deposition of monolayer transition metal dichalcogenides and their temperature dependent Raman spectroscopy studies. Nanoscale 8, 3008-3018 (2016).

25. Kandemir, A., Peeters, F. M. \& Sahin, H. Monitoring the effect of asymmetrical vertical strain on Janus single layers of MoSSe via vibrational spectrum. J. Chem. Phys. 149, 084707 (2018).

26. Taghizadeh, A., Leffers, U., Pedersen, T. G. \& Thygesen, K. S. A library of ab initio Raman spectra for automated identification of $2 \mathrm{D}$ materials. Nat. Commun. 11, 3011 (2020)
27. Lee, $C$. et al. Anomalous lattice vibrations of single-and few-layer $\mathrm{MOS}_{2}$. ACS Nano 4, 2695-2700 (2010).

28. Zhao, Y., Lee, H., Choi, W., Fei, W. \& Lee, C. J. Large-area synthesis of monolayer $\mathrm{MoSe}_{2}$ films on $\mathrm{SiO}_{2} / \mathrm{Si}$ substrates by atmospheric pressure chemical vapor deposition. RSC Adv. 7, 27969-27973 (2017).

29. Petrić, M. M. et al. Raman spectrum of Janus transition metal dichalcogenide monolayers WSSe and MoSSe. Phys. Rev. B 103, 035414 (2021).

30. Zhao, S. et al. A Se vacancy induced localized Raman mode in twodimensional $\mathrm{MoSe}_{2}$ grown by CVD. arXiv.1904.09789 (2019).

31. Yanase, T., Uehara, F., Naito, I., Nagahama, T. \& Shimada, T. Healing sulfur vacancies in monolayer $\mathrm{MoS}_{2}$ by high-pressure sulfur and selenium annealing: Implication for high-performance transistor. ACS Appl. Nano Mater. 3, 10462-10469 (2020).

32. Wang, L., Ji, X., Chen, F. \& Zhang, Q. Temperature-dependent properties of monolayer $\mathrm{MoS}_{2}$ annealed in an Ar diluted S atmosphere: an experimental and first-principles study. J. Mater. Chem. C. 5, 11138-11143 (2017).

33. Li, H. et al. Lateral growth of composition graded atomic layer $\operatorname{MoS}_{2(1-x)} \mathrm{Se}_{2 x}$ nanosheets. J. Am. Chem. Soc. 137, 5284-5287 (2015).

34. Pitthan, E., Gerling, E. R. F., Feijó, T. O., Radtke, C. \& Soares, G. V. Annealing response of monolayer $\mathrm{MoS}_{2}$ grown by chemical vapor deposition. ECS J. Solid State Sci. Technol. 8, P267-P270 (2019).

35. Zhu, S. \& Wang, Q. A simple method for understanding the triangular growth patterns of transition metal dichalcogenide sheets. AlP Adv. $\mathbf{5}$ 107105 (2015)

36. Raju, M., Wan, M., Sen, S. \& Jacob, C. Influence of chemical potential on shape evolution of $2 \mathrm{D}-\mathrm{MoS}_{2}$ flakes produced by chemical vapor deposition. Nanotechnology 32, 045301 (2021).

37. Zhang, G. et al. Shape-dependent defect structures of monolayer $\mathrm{MoS}_{2}$ crystals grown by chemical vapor deposition. ACS Appl. Mater. Interfaces 9 1763-1770 (2017).

38. Shin, D. H., Jang, C. W., Ko, J. S. \& Choi, S.-H. Enhancement of efficiency and stability in organic solar cells by employing $\mathrm{MoS}_{2}$ transport layer, graphene electrode, and graphene quantum dots-added active layer. Appl. Surf. Sci. 538, 148155 (2021).

39. Pan, Y. J. et al. Interfacial properties of monolayer $\mathrm{MoSe}_{2}$-metal contacts. J. Phys. Chem. C. 120, 13063-13070 (2016). 